\title{
Cystic angiomatosis skull lesion obliteration with neuroendovascular sclerotherapy as a unique treatment: case report
}

\author{
Guilherme B. F. Porto, MD, ${ }^{1}$ Cynthia T. Welsh, MD, ${ }^{2}$ M. Imran Chaudry, MBBS,${ }^{3}$ and \\ Ramin Eskandari, MS, MD1
}

Departments of ${ }^{1}$ Neurosurgery and ${ }^{2}$ Pathology and Laboratory Medicine, Medical University of South Carolina, Charleston; and ${ }^{3}$ Department of Neuroradiology and Neuroendovascular Surgery, Greenville Memorial Hospital, Greenville, South Carolina

\begin{abstract}
Cystic angiomatosis is a rare bone condition with complex presentation and difficult treatment. Current management strategies have poorly tolerated side effects and a low likelihood of disease eradication. The control of calvarial lesions that are symptomatic usually involves surgical excision and subsequent cranioplasty. This paradigm can present with a risk of morbidity and mortality depending on the anatomy of the lesion. Here, the authors present a novel approach to a difficult-to-treat occipital calvarial lesion directly overlying the transverse sinus, performing a small, partial-thickness craniectomy and alcohol sclerotherapy in a combined neurosurgery-neuroendovascular approach. At 3 years after treatment, the authors noted a complete, encouraging radiographic and clinical outcome.
\end{abstract}

https://thejns.org/doi/abs/10.3171/2020.3.PEDS2045

KEYWORDS cystic angiomatosis sclerotherapy; pediatric cystic angiomatosis; oncology

A BNORMAL angiogenesis in bony matrix has been implicated as the culprit in a myriad of related bone disorders, including cystic angiomatosis (CA). This extremely rare condition, described in adults and children, has a wide range of nonskeletal systemic involvement. ${ }^{1-6}$ First described in 1940 by Parsons and Ebbs, ${ }^{7}$ it is characterized by axial and appendicular skeletal hemangiomatous lesions frequently with multiple organ systems involved at presentation. ${ }^{8-10}$ The disease has a predilection for males and has a bimodal age distribution, with one peak manifesting during puberty and the other in middle age, ${ }^{11-14}$ suggesting both congenital and acquired etiologies. The most severe form of the disease, Gorham-Stout disease (GSD), presents with destruction and resorption of bone, leading to fractures, deformities, and even functional impairment. The two conditions are usually thought of as part of a spectrum, and their natural histories are vastly different. CA is an indolent form, and patients generally have medullary lesions that, although painful, tend not to progress once successfully treated. GSD, however, in- volves cortical bone, pathological fractures, and massive osteolysis, causing disability and metabolic derangements. ${ }^{5}$ The pathophysiology of osseous lesions has yet to be elucidated, although proliferation of endothelial-lined vessels within the bone has been implicated. ${ }^{15}$ Laboratory findings include an increase in alkaline phosphatase, as well as the bone marker osteoprotegerin, osteopenia, and interleukin- $6 .^{10,16}$ The treatment paradigm for CA and related conditions depends on disease extent, but excision, radiation therapy, and medical treatments such as bisphosphonates, interferon- $\alpha$, and calcitonin have all been described. ${ }^{17-20}$ Unfortunately, the location of lesions within the calvaria presents unique risks for surgery, radiation, and medical therapies. ${ }^{21}$ Lesions over large venous sinuses present increased risk for dural injury during surgery and subsequent cranioplasty, whereas radiation to the skull increases the risk for scatter into the brain. Further, radiation therapy, although usually $75 \%$ effective at local lesion control in GSD, has been shown to have minimal to no effect on CA lesions. $^{21-24}$ 
Aforementioned medical therapies also carry systemic side effects, which can be both unpleasant and damaging, including a range of conditions from flu-like symptoms to multiorgan system complications such as liver failure and central neurotoxicity as well as an ever-present risk of treatment failure. ${ }^{25-27}$ For this reason, finding alternative minimally invasive methods for directly treating lesions of the skull without local or systemic side effects would be beneficial. Ethanol sclerotherapy has a wide range of vascular applications, including percutaneous injections of vascular malformations, varicoceles, lymphatic malformations as well as parathyroid and thyroid tumor ablations. ${ }^{28}$ Considering that the nature of these lesions is endothelial in origin, we pursued a novel neuroendovascular sclerotherapy approach for a lesion directly over the transverse sinus in a pediatric patient. To our knowledge, no literature to date describes successful treatment with neuroendovascular sclerotherapy for a CA lesion.

\section{Case Report}

\section{History and Examination}

A 17-year-old white female with a diagnosis of CA had been initially evaluated by neurosurgery because of a new tender lesion in the right frontal region at age 15. At the time, imaging demonstrated multiple lesions in the calvaria including a left occipital lesion as well as right frontal and clival lesions, without any evidence of intracranial invasion or destruction of the bony inner table of the cranium. Systemic workup revealed splenomegaly as well as extensive appendicular skeleton involvement with lytic lesions seen in the ribs, L5 vertebral body, pelvis, and proximal femurs, concerning for Langerhans cell histiocytosis. She underwent a craniectomy for biopsy of a right frontal bony lesion, and pathology reported fibrous dysplasia due to its significant reactive bone formation. Serial imaging demonstrated enlargement of the remaining lesions, inconsistent with the aforementioned diagnosis. Resection of a second lesion in the right parietal bone was performed, and after multiple independent pathological evaluations, the patient was diagnosed with CA (Fig. 1). She was followed by pediatric neurosurgery and underwent multiple craniectomies with resorbable epidural plate and hydroxyapatite bone cement-augmented cranioplasty for both new and progressing prior calvarial lesions as well as resection of a femoral lesion with placement of three cannulated screws into the femoral head and neck for stabilization. Planned infusions of zoledronic acid (Reclast, Novartis Pharmaceuticals) at $0.025 \mathrm{mg} / \mathrm{kg}$ were aborted after one dose because the patient demonstrated intolerance to this therapy. Discussion of adjuvant radiation and medical therapies included multiple medical centers, and the family declined to pursue these for fear of potential side effects and complications. The patient continued routine pediatric neurooncology, hematology, and endocrinology follow-up.

Despite all of the above interventions, she presented with a new palpable left occipital calvarial lesion without intracranial extension, located directly over the left transverse sinus (Fig. 2A). The patient and her family were counseled on yet another craniectomy including the risks for sinus injury, hemorrhage, and stroke. They desperately wished for an alternative therapy option. After discus-

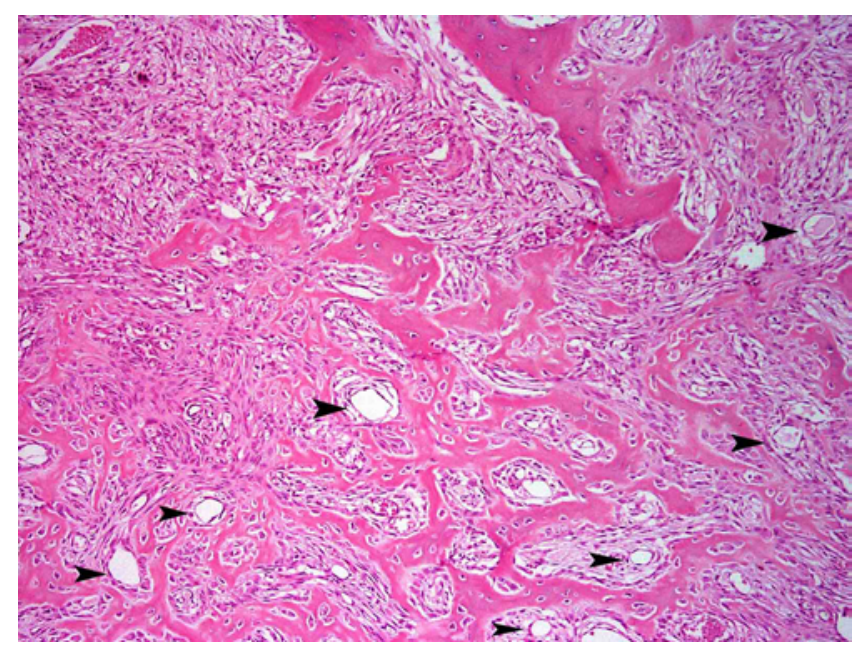

FIG. 1. Photomicrograph showing that an increase in thin-walled capillary-size vessels (arrowheads) has caused reactive bone spicules, fibrosis, and an osteoblastic reaction. $\mathrm{H} \& \mathrm{E}$, original magnification $\times 5$. Figure is available in color online only.

sion between the pediatric neurosurgeon and the neuroendovascular radiologist, the idea of neuroendovascular sclerotherapy was entertained. Given the vascular nature of CA, with imaging consistently demonstrating a highly enhancing central portion, the consensus was to perform a partial-thickness twist drill burr hole procedure for endovascular access and injection of a sclerosing alcohol agent. A diagnostic cerebral and extracranial arteriogram and venogram were obtained prior to sclerotherapy to ensure no anomalous extracranial-intracranial anastomoses and no intracranial vascular supply to the lesion (Fig. 3A-C).

\section{Operation}

The patient was intubated, and the procedure was performed in the neuroendovascular suite with pediatric neurosurgery and neurointerventional radiology. A small incision was made over the vertex of the palpable lesion, and a partial-thickness twist drill burr hole procedure through the cortical bone was performed slowly in order to not penetrate the inner table. Direct access to the cancellous trabeculated CA lesion was confirmed using a 23-gauge butterfly needle placed in the drill track, and intraoperative Dyna CT was performed to ensure partial-thickness insertion without violation of the inner table. Next, $1 \mathrm{ml}$ of iohexol contrast medium (Omnipaque, GE Healthcare) was introduced into the lesion under fluoroscopy to delineate the size and volume of the lesion (Fig. 3D and E). There was no systemic venous drainage and no contrast extravasation intracranially. One milliliter of 98\% (196 proof) dehydrated alcohol was injected, and contrast injection under fluoroscopic guidance afterward demonstrated slow accumulation of contrast with no systemic drainage. At this point the butterfly needle was removed from the lesion, and the skin was closed and dressed with a small gauze pressure dressing and bandage.

\section{Postoperative Course}

The patient emerged from anesthesia without complications. She was discharged home the same day. MRI with 
Porto et al.
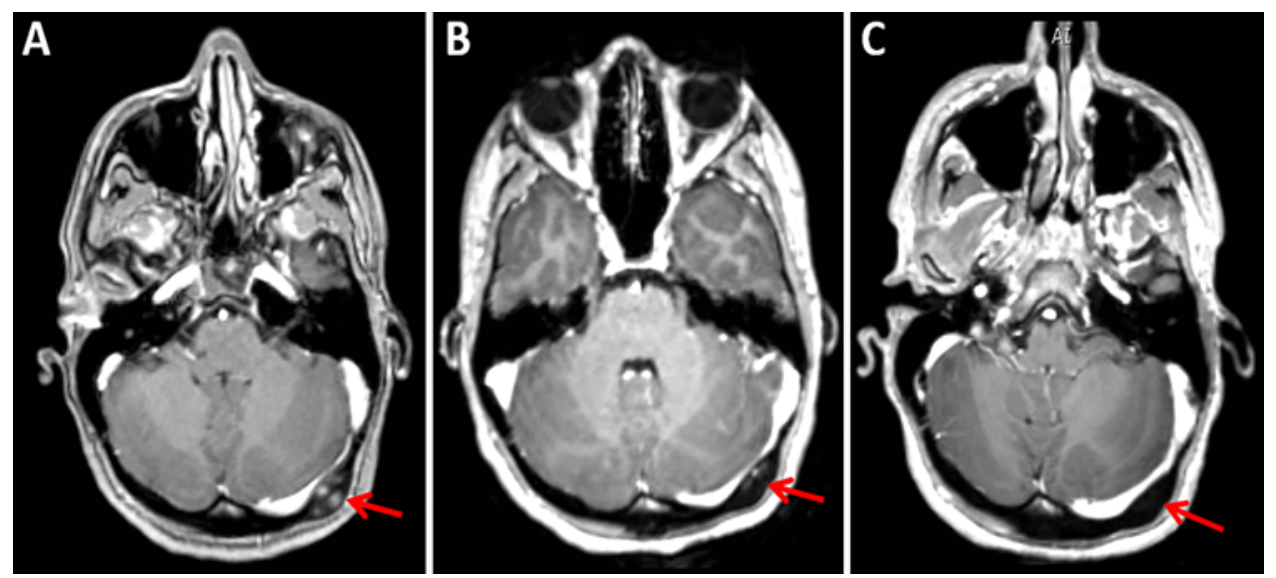

FIG. 2. A: Preoperative axial MRI with contrast demonstrated a left occipital vascular bone lesion (arrow) with T1 intrinsic hyperintense signal and no enhancement, measuring $2.8 \times 1.2 \times 1.5 \mathrm{~cm}$. B: Axial MRI with contrast demonstrated lesion size reduction (arrow) at the 1-year follow-up. C: Repeat MRI with contrast demonstrated complete devascularization of the lesion (arrow), with no evidence of intrinsic T1 hyperintense signal and no further growth at the 3-year follow-up. Figure is available in color online only.

contrast was performed intermittently until the final follow-up at 3 years postsclerotherapy, which demonstrated complete devascularization of the lesion, with no evidence of intrinsic T1 hyperintense signal or further growth (Fig. $2 \mathrm{~B}$ and $\mathrm{C})$.
At the orthopedic surgery follow-up 2 years postsurgical fixation, her hardware was stable and there were sclerotic changes compatible with healing, but no worsening of the lytic lesions. A full skeletal survey demonstrated a stable L5 lytic lesion and other small bony lesions
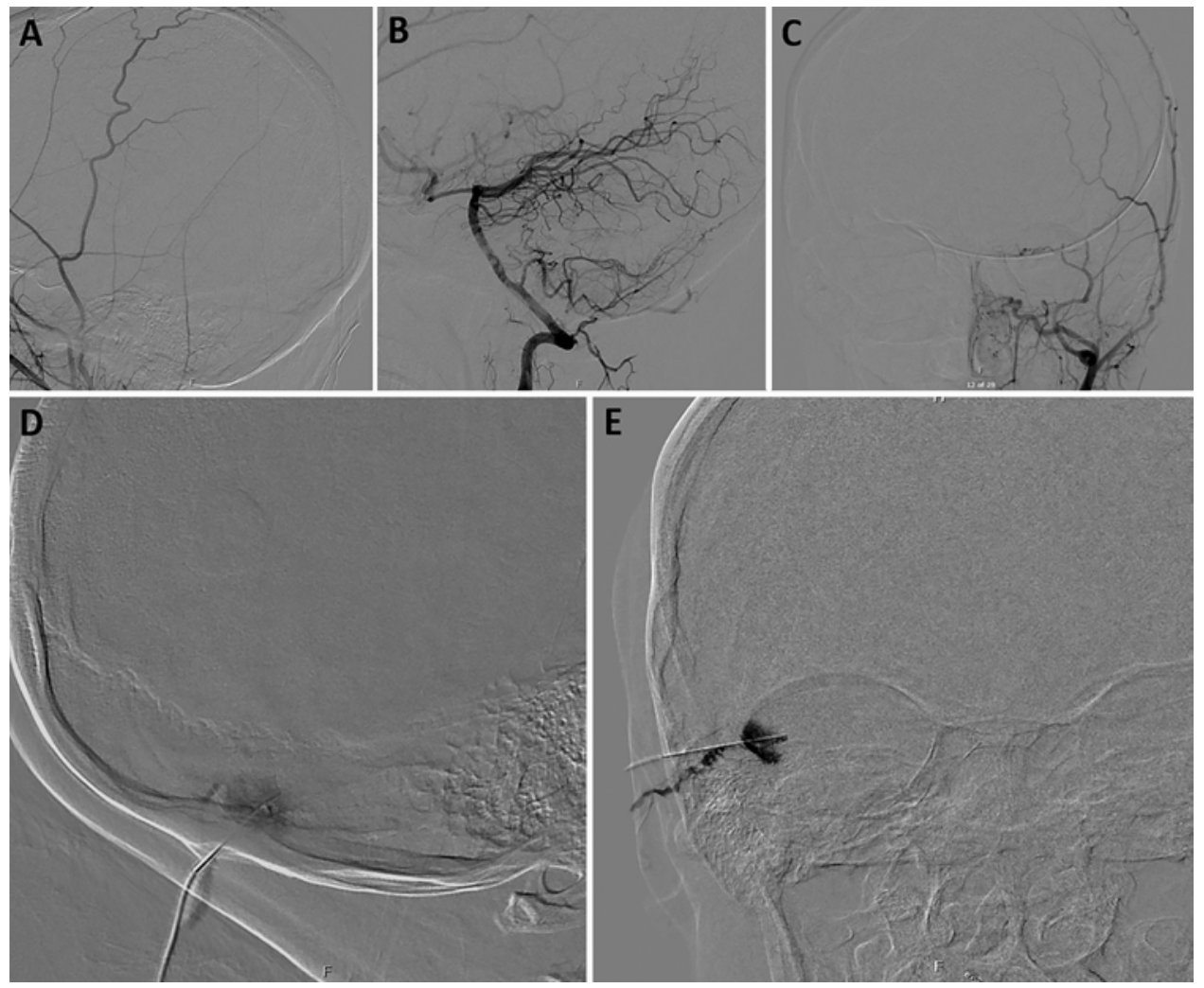

FIG. 3. Digital subtraction angiogram of the left external carotid artery, lateral (A) and anterior-posterior (B), demonstrating no vascular communication with the occipital lesion. Left vertebral artery angiogram (C) demonstrating no intracranial vascular supply to the occipital lesion. Contrast injection ( $D$ and $E$ ) via a 23-gauge butterfly needle demonstrating a vascular trabeculated bony lesion without dissemination to intra- or extracranial vasculature. 
throughout appendicular skeleton. Because most of these lesions were asymptomatic and because of the patient's poor tolerance of medical therapy and the family's wishes to avoid radiation therapy, conservative management was chosen unless lesion progression was observed. From a medical standpoint, the patient's endocrinology follow-up showed stable metabolic values, including interleukin-6, urine, and blood calcium values, without evidence of systemic progression.

\section{Discussion}

Cystic angiomatosis is a complex multisystem disease with calvarial lesions that often cause pain and morbidity. Sclerotherapy for CA and related vascular disorders, including GSD and lymphangiomatosis, has risen in popularity as a viable treatment option over the years. The procedure is commonly used in patients who have a large vascular lesion and no appropriate or safe surgical or medical treatment option. Resection, although possibly curative if complete, is often plagued by young patients who undergo large surgeries with unacceptable scarring, as well as high risks for infection, disease recurrence, and surrounding tissue injury including neural or vascular damage that could lead to long-lasting morbidity and high mortality. Sclerotherapy, on the other hand, is generally well tolerated and has few contraindications, that is, bony disease with intracranial extension, an arteriovenous fistula with contrast extravasation into normal brain vasculature or a major sinus, and the presence of coagulation disorders or anticoagulation state.

Patients frequently undergo multiple cranial surgeries for biopsy and lesion resection with a high risk of recurrence. Medical treatment is poorly understood because of the limited literature, and although bisphosphonates seem to be the treatment of choice, this class of drugs is usually poorly tolerated and appropriate dosing is unknown, especially in the pediatric population. We describe the first published treatment of a calvarial lesion with a partialthickness needle entry over the transverse sinus and direct site injection of dehydrated alcohol as a sclerotherapeutic agent in a hybrid neuroendovascular suite with the assistance of a neurointerventionalist. The patient recovered well and had same-day discharge, and imaging revealed size reduction at 1 year and obliteration of the lesion at the 3-year follow-up. More patients need to be treated with this procedure, and long-term follow-up is needed to validate our experience; however, considering the low morbidity of the procedure and our success with this patient, this treatment choice for CA lesions requiring intervention may obviate the need for extensive craniectomies and cranioplasties. Alcohol sclerotherapy could change the treatment paradigm for bony vascular conditions in the CA spectrum as well as a wide range of vascular malformation pathologies, potentially becoming first-line therapy for symptomatic lesions. Considering the inherent risks with surgical intervention, this minimally invasive and relatively low-risk intervention must be disseminated and further investigated in an effort to bring better treatment options to patients affected by these rare conditions.

\section{References}

1. Bauschard MJ, Hatch JL, Liogier-Weyback LE, et al. Intracranial complications from temporal bone cystic angiomatosis. Otol Neurotol. 2017;38(2):e1-e2.

2. Jacobs JE, Kimmelstiel P. Cystic angiomatosis of the skeletal system. J Bone Joint Surg Am. 1953;35-A(2):409-420.

3. Kumar V, Meghal T, Sun YH, Huang Y. Systemic cystic angiomatosis mimicking metastatic cancer: a case report and review of the literature. Case Rep Med. 2017;2017:5032630.

4. Marraoui W, Michel JL, Kemeny JL, Soubrier M. Imaging features of systemic cystic angiomatosis. Diagn Interv Imaging. 2015;96(11):1211-1213.

5. Najm A, Soltner-Neel E, Le Goff B, et al. Cystic angiomatosis, a heterogeneous condition: four new cases and a literature review. Medicine (Baltimore). 2016;95(43):e5213.

6. Seckler SG, Rubin H, Rabinowitz JG. Systemic cystic angiomatosis. Am J Med. 1964;37:976-986.

7. Parsons LG, Ebbs JH. Generalized angiomatosis presenting the clinical characteristics of storage reticulosis: with some observations on the reticulo-endothelioses. Arch Dis Child. 1940;15(83):129-158.

8. Deveci M, Inan N, Corapçıŏlu F, Ekingen G. Gorham-Stout syndrome with chylothorax in a six-year-old boy. Indian $J$ Pediatr. 2011;78(6):737-739.

9. Dos Anjos CS, Borges RMC, Chaves AC, et al. Cystic angiomatosis, pleural effusion and multiple bone lesions mimicking a metastatic malignant neoplasia: a case report. J Med Case Reports. 2019;13(1):265.

10. Marcucci G, Masi L, Carossino AM, et al. Cystic bone angiomatosis: a case report treated with aminobisphosphonates and review of the literature. Calcif Tissue Int. 2013;93(5):462-471.

11. Boyle WJ. Cystic angiomatosis of bone. A report of three cases and review of the literature. J Bone Joint Surg Br. 1972;54(4):626-636.

12. Devaney K, Vinh TN, Sweet DE. Skeletal-extraskeletal angiomatosis. A clinicopathological study of fourteen patients and nosologic considerations. J Bone Joint Surg Am. 1994;76(6):878-891.

13. Levey DS, MacCormack LM, Sartoris DJ, et al. Cystic angiomatosis: case report and review of the literature. Skeletal Radiol. 1996;25(3):287-293.

14. Sferopoulos NK, Anagnostopoulos D, Webb JK. Cystic angiomatosis of bone with massive osteolysis of the cervical spine. Eur Spine J. 1998;7(3):257-259.

15. Schajowicz F, Aiello CL, Francone MV, Giannini RE. Cystic angiomatosis (hamartous haemolymphagiomatosis) of bone. A clinicopathological study of three cases. J Bone Joint Surg Br. 1978;60(1):100-106.

16. Brower AC, Culver JE Jr, Keats TE. Diffuse cystic angiomatosis of bone. Report of two cases. Am J Roentgenol Radium Ther Nucl Med. 1973;118(2):456-463.

17. Avelar RL, Martins VB, Antunes AA, et al. Use of zoledronic acid in the treatment of Gorham's disease. Int J Pediatr Otorhinolaryngol. 2010;74(3):319-322.

18. Mignogna MD, Fedele S, Lo Russo L, Ciccarelli R. Treatment of Gorham's disease with zoledronic acid. Oral Oncol. 2005;41(7):747-750.

19. Moschetta M, Di Pietro G, Ria R, et al. Bortezomib and zoledronic acid on angiogenic and vasculogenic activities of bone marrow macrophages in patients with multiple myeloma. Eur J Cancer. 2010;46(2):420-429.

20. Plontke S, Koitschev A, Ernemann U, et al. Massive Gorham-Stout osteolysis of the temporal bone and the craniocervical transition [in German]. HNO. 2002;50(4):354-357.

21. Pavanello M, Piatelli G, Ravegnani M, et al. Cystic angiomatosis of the craniocervical junction associated with Chiari I malformation: case report and review of the literature. Childs Nerv Syst. 2007;23(6):697-700. 
22. Dellinger MT, Garg N, Olsen BR. Viewpoints on vessels and vanishing bones in Gorham-Stout disease. Bone. 2014;63:4752 .

23. Gramiak R, Ruiz G, Campeti FL. Cystic angiomatosis of bone. Radiology. 1957;69(3):347-353.

24. Gutierrez RM, Spjut HJ. Skeletal angiomatosis: report of three cases and review of the literature. Clin Orthop Relat Res. 1972;85(85):82-97.

25. Devlin RD, Bone HG III, Roodman GD. Interleukin-6: a potential mediator of the massive osteolysis in patients with Gorham-Stout disease. J Clin Endocrinol Metab. 1996;81(5):1893-1897.

26. Hagberg H, Lamberg K, Aström G. Alpha-2b interferon and oral clodronate for Gorham's disease. Lancet. 1997;350(9094):1822-1823.

27. Hammer F, Kenn W, Wesselmann U, et al. Gorham-Stout disease-stabilization during bisphosphonate treatment. $J$ Bone Miner Res. 2005;20(2):350-353.

28. Albanese G, Kondo KL. Pharmacology of sclerotherapy. Semin Intervent Radiol. 2010;27(4):391-399.

\section{Disclosures}

The authors report no conflict of interest concerning the materials or methods used in this study or the findings specified in this paper.

\section{Author Contributions}

Conception and design: Porto, Eskandari. Acquisition of data: all authors. Analysis and interpretation of data: Porto, Welsh, Eskandari. Drafting the article: Porto. Critically revising the article: all authors. Reviewed submitted version of manuscript: Porto. Study supervision: Eskandari.

\section{Correspondence}

Guilherme B. F. Porto: Medical University of South Carolina, Charleston,SC.porto@musc.edu. 